\title{
Biomarkers for Antidepressant Efficacy of Electroconvulsive Therapy: An Exploratory Cerebrospinal Fluid Study
}

Laura Kranaster a, Carolin Hoyer ${ }^{\text {b }}$, Suna S. Aksay a, J. Malte Bumb c , Norbert Müller ${ }^{d}$, Peter Zill ${ }^{d}$, Markus J. Schwarz ${ }^{e}$, Christoph Janke ${ }^{f}$, Manfred Thiel ${ }^{f}$, Beat Lutz 9 ,

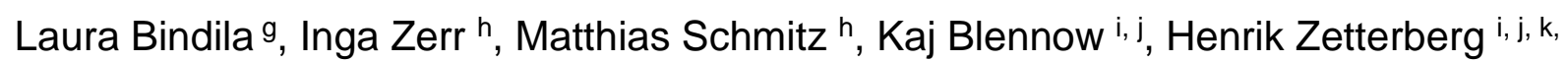
', Dieter Haffner ${ }^{m}$, Maren Leifheit-Nestler ${ }^{m}$, Alexander Sartorius ${ }^{a}$

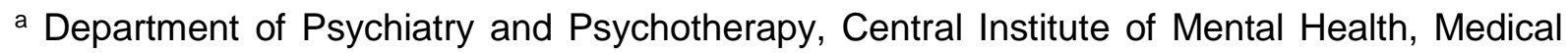
Faculty Mannheim / Heidelberg University, Mannheim, Germany

${ }^{\mathrm{b}}$ Department of Neurology, University Medical Centre Mannheim, Mannheim, Germany

${ }^{\mathrm{C}}$ Department of Addictive Behavior and Addiction Medicine, Central Institute of Mental Health, Medical Faculty Mannheim/Heidelberg University, Mannheim, Germany

d Department of Psychiatry and Psychotherapy, Ludwig Maximilian University Munich, Germany

${ }^{\text {e }}$ Department of Laboratory Medicine, Ludwig Maximilian University Munich, Germany

${ }^{f}$ Department of Anesthesiology and Critical Care Medicine, University Medical Centre Mannheim, Mannheim, Germany

${ }^{9}$ Institute for Physiological Chemistry, University Medical Center Mainz of the Johannes Gutenberg University Mainz, Germany

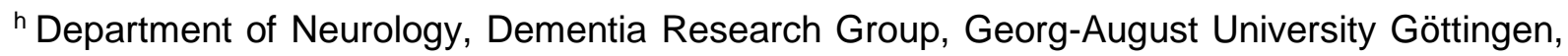
Germany

' Department of Psychiatry and Neurochemistry, Institute of Neuroscience and Physiology, the Sahlgrenska Academy at the University of Gothenburg, Mölndal, Sweden

j Clinical Neurochemistry Laboratory, Sahlgrenska University Hospital, Mölndal, Sweden

${ }^{k}$ Department of Molecular Neuroscience, UCL Institute of Neurology, London, UK 
' UK Dementia Research Institute at UCL, London, UK.

${ }^{m}$ Department of Pediatric Kidney, Liver and Metabolic Diseases, Hannover Medical School, Hannover, Germany

\section{Corresponding author:}

Laura Kranaster

Department of Psychiatry and Psychotherapy

Central Institute of Mental Health, Medical Faculty Mannheim / Heidelberg University

J5, 68159 Mannheim, Germany

Phone: +49 621 1703-0, Fax: +49 621 1703-1205

E-Mail: laura.kranaster@zi-mannheim.de 
Abstract

IN PREPARATION 


\section{Introduction}

Electroconvulsive therapy $(E C T)$ is an effective and safe treatment option for specific forms of depression, such as treatment-resistant depression, psychotic depression or melancholia in both, uni- and bipolar affective disorder [1, 2], with response rates usually ranging around $70 \%$. Despite this remarkable efficacy even in highly treatment-resistant depression, there is still a considerable group of non-responders to ECT. Therefore, it is of high clinical interest to identify factors or markers that could provide information prior to treatment on whether the individual patient will respond to ECT or not.

Previous studies have provided a large body of evidence about potentially prognostic demographic or clinical factors such as age, bipolarity, family history of depression or comorbid axis II disorder that might contribute to the risk of non-response to ECT [3, 4]. Others tried to elucidate whether a set of ictal parameters might predict the clinical outcome $[5,6]$. More recently, several imaging markers were successfully used to predict the outcome to ECT in major depression, for example the baseline level of higher BOLD signal fluctuations of the subcallosal cingulate cortex [7], the subgenual cingulate volume [8] and several gray matter regions [9].

A few blood-based candidate biomarkers for ECT treatment response have been identified, including vascular endothelial growth factor [10] and homovanillic acid [11]. In contrast to markers from blood that might not be central nervous system (CNS)-specific, markers measured in the cerebrospinal fluid (CSF) could reflect cerebral processes much more directly. However, biomarkers in the CSF that could predict outcome to ECT in patients with major depression have not been identified yet.

We recently ran a study in which CSF samples before and after a course of ECT in patients with severe depression were collected. Thus, we had the opportunity to analyse several markers from different areas, such as lipids (endocannabinoids and sphingolipids), cytokines, elements from the kynurenine pathway and different markers of neurodegeneration. In this 
study, we analysed these biomarkers in patients with depression to examine if there were correlations of their baseline CSF levels before ECT with the clinical outcome of ECT.

\section{Methods}

\section{Patients}

Our prospective study was approved by the appropriate ethics committee and was performed in accordance with The Code of Ethics of the World Medical Association (Declaration of Helsinki). Written informed consent was obtained from all participants before enrolment. The study took place from 2012 to 2014 at the Department of Psychiatry at the Central Institute of Mental Health in Mannheim, Germany. Inclusion criteria were a present treatment resistant depressive episode (defined as failure to achieve response or remission to at least two proven antidepressant trial with adequate dosing and duration [12]) within the context of a diagnosis of either major depressive disorder or bipolar disorder according to DSM-IV, age above 18 years and the clinical decision for an ECT treatment. Exclusion criteria were substance-related disorders including tobacco and alcohol, the lifetime diagnosis of schizophrenia, any signs of an on-going inflammation process such as leucocytosis, elevated acute phase proteins or fever, the intake of any medication with known immunomodulatory properties such as nonsteroidal anti-inflammatory drugs or glucocorticoids and the reported use of $\Delta^{9}$ tetrahydrocannabinol (THC) twelve months before the study or detection of THC in urine drug screen. The patients continue their prior psychotropic medication during the ECT treatment. The Hamilton Depression Rating Scale (HDRS; 21 items version) was used to assess the severity of depression before and after the ECT in each patient. Response was defined as a reduction of at least $50 \%$ in symptoms measured by the HDRS. The Mini-Mental-State Examination (MMSE) was used to assess the gross cognitive performance prior to treatment.

\section{ECT treatment}

Right unilateral brief pulse ECT was performed with a Thymatron IV device (Somatics, LLC. Lake Bluff, IL, USA), s-ketamine was used as the anaesthetic substance $(\sim 1.0 \mathrm{mg} / \mathrm{kg})[13,14]$ 
and succinylcholine for muscle relaxation $(\sim 1.0 \mathrm{mg} / \mathrm{kg})$. Seizure threshold in all patients was titrated at the initial session and dosing at subsequent treatments was given at $>2.5$ times threshold. The energy was subsequently increased if patients did not respond clinically or if seizures were insufficient during the ECT course. Two or three ECT sessions per week were performed. All patients started with unilateral stimulation, in the case of non-response, it was possible to switch to bilateral stimulation as a decision of the ECT supervisor.

\section{Sampling}

Before the first ECT session and between one and seven days after the last ECT session, all CSF samples were drawn at around 9:00 am. The patients were in a non-fasting status. They had a light breakfast at 8:00 am. Lumbar punctures were performed in the sitting position according to standardized procedure. All samples were taken, aliquoted and transferred at $80^{\circ} \mathrm{C}$ within 30 min either in glass vials (Hycultec, Beutelsbach, Germany) for the analyses of the lipids, in polypropylene tubes for the analyses of the markers of neurodegeneration or in protein LoBind tubes (Eppendorf, Germany) for all other substances. The samples were stored at $-80^{\circ} \mathrm{C}$ and were never thawed or refrozen prior to the presented analyses.

\section{CSF and serum laboratory analyses}

Baseline and follow-up samples were analyzed on one occasion on the same plates using the same batch of reagents.

\section{$\underline{\text { Tau proteins and } \beta \text {-amyloids }}$}

All samples were measured at the National TSE (Transmissible Spongiform Encephalopathies) Reference Centre (Göttingen, Germany). CSF levels of $A \beta_{1-40}, A \beta_{1-42}$, total tau and its phosphorylated isoform were determined with commercially available enzymelinked immunosorbent assay (ELISA) kits, described elsewhere [15].

\section{$\underline{\text { Neurogranin }}$}


All samples were measured at the Clinical Neurochemistry Laboratory at Sahlgrenska University Hospital. CSF $\mathrm{Ng}$ concentration was measured by enzyme-linked immunosorbent assay (ELISA), essentially as described [16], using the anti- $\mathrm{Ng}$ antibody pair $\mathrm{Ng} 2$ and $\mathrm{Ng} 22$. Intra-assay coefficients of variation were below $10 \%$.

\section{Endocannabinoids}

All samples were measured at the Lipidomics/Mass Spectrometry Facility at the Institute for Physiological Chemistry at the University Medical Center Mainz. Endocannabinoids were qualitatively and quantitatively analyzed using liquid chromatography/ multiple reaction monitoring (LC/MRM) according to the procedures described elsewhere [17]. Levels of both AEA and 2-AG were measured, as well as levels of arachidonic acid (AA), from which both endocannabinoids are derived.

\section{Elements of the innate immune system}

CSF concentrations were measured as instructed by the manufacturer, using sandwich ELISA kits for interleukin-6 (IL-6), neopterin, soluble CD14 (sCD14), soluble CD163 (sCD163), macrophage migration inhibitory factor (MIF) and monocyte chemotactic protein 1 (MCP-1) as described elsewhere [18].

\section{Metabolites of the Kynurenine Pathway}

CSF levels of kynurenine (KYN), kynurenic acid (KYNA), picolinic acid (PIC), quinolinic acid (QUIN), tryptophan (TRY), 3-hydroxykynurenine (3-HK) were determined using a highperformance liquid chromatography kit. For further details see Supplementary Methods. From these values, the kynurenine-to-tryptophan ratio (KYN/TRY) was calculated.

\section{Sphingolipids}

BESCHREIBUNG AUS HEIDELBERG FEHLT NOCH

sKlotho 
All samples were measured at the Department of Pediatric Kidney, Liver and Metabolic Diseases, Hannover Medical School, Hannover, Germany. The concentration of Klotho in CSF was quantified using a human Klotho enzyme-linked immunosorbent assay (ELISA) kit measuring the entire soluble $\alpha$-Klotho protein secreted in the systemic circulation after its release from the cell membrane via shedding (cleaved Klotho) or alternative splicing (secreted Klotho) with an assay sensitivity of $6.15 \mathrm{pg} / \mathrm{mL}$ (Immuno-Biological Laboratories Co., Ltd., Gunma, Japan).

\section{Statistical analyses}

Statistics were performed using STATA® (StataCorp, Texas 77845, USA, version 11) at a significance level $\leq 0.05$. Normality was examined using the Shapiro-Wilk and data were logtransformed when necessary. No correction for multiple testing was performed to avoid type II errors in this study with its small sample size. In line with that, results with significance levels between 0.05 and 0.1 were additionally reported as trend observations. Spearman's correlation $\left(r_{s}\right)$ was used to determine the strength and direction of the relationship between the concentration of the different markers and either the extent of depressive symptoms at baseline or the reduction of depressive symptoms during ECT or possible moderators. Linear regression was used to estimate which single marker could predict the reduction of the HDRS score during ECT and multiple regression analysis with stepwise inclusion was used to identify models, in which more than one marker could add significantly to the prediction. Group differences between responder and non-responder were examined with the Wilcoxon ranksum test. Logistic regression (responder vs. non-responder) was not be performed due to the small sample size.

\section{Results}

We included 12 patients into the study, who underwent a completed course of ECT and lumbar puncture before and thereafter. In general, ECT was effective with a reduction of the mean initial HDRS from $29.9 \pm 6.6$ to $9.0 \pm 5.2$ after the final session $(p<0.001)$. Ten patients $(83.3 \%)$ could be classified as treatment responder, whereas two patients (16.7\%) were considered as 
non-responders to ECT. Three patients (two females, one male, mean age: $75.7 \pm 6.4$ ) had the clinical diagnosis of an Alzheimer's disease (AD) additional to their current severe depressive episode. Demographic and clinical features are shown in Table 1.

\section{Absolute reduction of depressive symptoms}

CSF baseline levels of AEA ( $\left.r_{s}=0.65 ; p=0.029\right), A \beta_{1-40}\left(r_{s}=0.64 ; p=0.025\right)$, tau protein $\left(r_{s}=0.68\right.$; $\mathrm{p}=0.015)$ and its phosphorylated isoform $\left(r_{s}=0.58 ; p=0.049\right), \mathrm{Ng}\left(r_{s}=0.64 ; p=0.024\right), s C D 14$ $\left(r_{s}=0.82 ; p=0.0021\right), K Y N\left(r_{s}=0.64 ; p=0.036\right)$ and $\mathrm{KYN} / \mathrm{TRY}\left(r_{s}=0.63 ; p=0.038\right)$ positively correlated with the absolute reduction of depressive symptoms during ECT. At trend level, we found this correlation also for CSF levels of IL-6 $\left(r_{s}=0.56 ; p=0.072\right)$, neopterin $\left(r_{s}=0.54\right.$; $p=0.087), \operatorname{sCD} 163(r s=0.54 ; p=0.084), P I C\left(r_{s}=0.68 ; p=0.062\right)$, QUIN $\left(r_{s}=0.67 ; p=0.070\right)$, phosphatidylcholines $\left(r_{s}=0.58 ; p=0.080\right)$ and the total amount of sphingolipids $\left(r_{s}=0.58\right.$; $p=0.080)$ (Figure 1-4). Age $(p=0.79)$, MMSE at baseline $(p=0.39)$, clinical AD diagnosis $(p=0.12)$ and sex $(p=0.44)$ were not associated with reduction of depressive symptoms during ECT. A summary of the significant findings is presented in Table 2.

Linear regression models established that all, $\operatorname{sCD} 14(F(1,9)=18.91 ; p=0.002)$, tau $(F(1$, $10)=9.3 ; p=0.013)$ and its phosphorylated isoform $(F(1,10)=9.49 ; p=0.012)$ (Figure $x)$ could statistically significantly predict the absolute reduction of depressive symptoms. However, no multiple regression model could be found that predicted this absolute reduction better than any single marker in the linear regression analyses.

\section{Relative reduction of depressive symptoms}

Correlations with the relative reduction of depressive symptoms during ECT were found for $\operatorname{sCD} 14\left(r_{s}=0.65 ; p=0.032\right), P I C\left(r_{s}=0.79 ; p=0.021\right)$ and $K Y N\left(r_{s}=0.61 ; p=0.047\right)$ and additional for KYNA $\left(r_{s}=0.54 ; p=0.086\right)$ and the kynurenine-to-tryptophan ratio $\left(r_{s}=0.56 ; p=0.076\right)$ at a statistical trend level of significance. We found no correlation with potential confounders such as age $(p=0.51)$, MMSE at baseline $(p=0.49)$ or the clinical AD diagnosis $(p=0.33)$. However, at trend level, females had a higher relative HDRS reduction by ECT than men ( $p=0.057)$. 
A linear regression model established that PIC $(F(1,6)=6.56 ; p=0.043)$ could statistically significantly predict the absolute reduction of depressive symptoms. Additionally, combining PIC and KYN in a multiple regression model predicted the relative reduction of depressive symptoms with both variables added statistically significantly to the prediction $(F(1,9)=27.8$; $\mathrm{p}=0.0020)$.

\section{Responders vs. Non-Responder}

We found a difference in the baseline CSF levels of $K Y N(z=2.1 ; p=0.034)$ in responders compared to those patients, who did not show a response to the treatment. At trend level, we found this correlation also for CSF levels of neurogranin $(z=2.0 ; p=0.052)$, tau protein $(z=1.7$; $p=0.086)$ and its abnormal phosphorylated isoform $(z=2.0 ; p=0.052), \operatorname{sCD} 163 \quad(z=1.7$; $p=0.099)$, kynurenic acid $(z=1.7 ; p=0.096)$ and PIC $(z=1.7 ; p=0.096)$. Age $(p=0.39)$, MMSE at baseline $(p=1.00)$, the clinical AD diagnosis $(p=0.42)$ or sex $(p=0.33)$ were not associated with the outcome parameter "response to ECT", defined as a reduction of at least $50 \%$ in symptoms measured by the HDRS.

\section{Discussion}

This is the first study that estimated the antidepressant therapy-outcome of ECT based on biomarker profiles in CSF at baseline, which could be a very interesting approach for future applications of biomarkers. We found a variety of markers from different areas that were correlated with either absolute or relative reduction of the HDRS that could be observed during a course of ECT or with the status responders vs. non-responders after ECT.

Markers of neurodegeneration, the innate immune system and metabolites of the kynurenine pathway were those that correlated the most with the antidepressant efficacy. In terms of neurodegeneration tau protein, its abnormal phosphorylated isoform, $A \beta_{1-40}$ and neurogranin, all known to be highly correlated or associated with each other [19] were correlated with any form of therapeutic effect, independent of confounders such as age or the presence of a clinical diagnosis of $A D$. A similar relationship between those markers and response to any 
antidepressant efficacy has not been reported yet and should be investigated in more detail in future studies. The baseline CSF concentrations of the metabolites of the kynurenine pathway, KYN, KYNA, the kynurenine-to-tryptophan ratio, PIC and QUIN were all positively correlated with the antidepressant effect of the ECT in those severely depressed patients. These findings are in general in line with the proposed involvement of the kynurenine pathway in depression [20-22]. Concerning the response to ECT, two recent studies that analysed the possible correlation to tryptophan metabolites in plasma did not observe such a correlation [23, 24], but no such studies has been carried out with analysis of the CSF, yet. Based on the relative abundance on the relationship between the immune system and different aspects of depression [25-27], it is not surprising that the baseline CSF levels of SCD14 and to a lesser extent of IL-6, neopterin and SCD163 - all involved primarily in the innate immune response [18] were positively correlated with the antidepressant outcome of ECT. Interestingly those three complex systems are tightly associated with each other: Changes of ( $p-)$ tau protein and $A \beta$ within Alzheimer's disease (AD) are known to be associated with alterations in the metabolites of the kynurenine pathway [28] and inflammation [29], whereas the kynurenine pathway links inflammation and the immune system to the pathophysiology of depression [21, $30,31]$ and inflammation is seen in both, depression and $A D$ with dysfunctional $A \beta$ and tau [32]. Our findings point towards an involvement of these complex systems in the antidepressant mechanism of ECT. To complement these modes to the established pathomechanisms of ECT, this involvement has to be explored in a more detailed way.

For lipids, the initial CSF levels of the endocannabinoid AEA and of elements of the sphingolipids were associated with antidepressant efficacy during ECT. Those markers have not been investigated concerning the issue of antidepressant outcome prediction yet, but at least our group recently reported that CSF AEA levels are increased by ECT and that those levels were positively correlated with the number of ECT session that were performed in each individual patient [33]. This finding corroborates a growing body of - primarily preclinical evidence that suggests an involvement of the endocannabinoid system in the pathophysiology of depression [34] and asks especially for more studies about the relationship between 
depression/antidepressant action and the endocannabinoid system in human. Sphingolipids, especially ceramides have already been linked with depression [35, 36], thus the results at a trend level significance could be cautiously integrated into this concept.

\section{Limitations}

The small sample size is probably the major limitation of our study. The samples were taken from a study, in which the patients were asked to consent to lumbar puncture twice - before and after ECT, therefore the recruitment was extremely difficult. Nonetheless, the sample size was large enough to identify larger effects and at least suggesting more moderate effects as well. Thus, these positive results from our small sample are promising to be replicated in future studies. Surely, we cannot exclude that with a larger sample size, smaller effects of other markers could have been unveiled. A further limitation in respect to the small sample size is that no adjustment for possible confounders such as age, sex or BMI could be performed. Based on the clinical reality, the study was only possible to conduct with our patients continuing some of their psychotropic medication that consisted of antidepressants, antipsychotics, benzodiazepines and lithium during the ECT treatment. In our cases, the medication in each patient was at least kept constant during the ECT course. Due to the design of our study, it is not possible to unmask any causal relationship between ECT and the identified CSF markers that correlated with the antidepressant efficacy. However, it is at least tempting to assume that the most efficient known antidepressant biological method, namely ECT works mostly efficient, when biological markers that might reflect any part of the pathway of depression are abnormal. In summary, our CSF study was primarily meant to identify markers that indicate whether the therapy option of ECT in patients with severe depression might be successful. Based on these markers, further research regarding the mechanism of ECT and personalized antidepressant therapy is suggested, but of course, treatment decisions for the individual must not be made based on our preliminary findings. 


\section{References}

1. Schoeyen HK, Kessler U, Andreassen OA, Auestad BH, Bergsholm P, Malt UF, Morken G, Oedegaard KJ, Vaaler A (2015) Treatment-resistant bipolar depression: A randomized controlled trial of electroconvulsive therapy versus algorithm-based pharmacological treatment. Am J Psychiatry 172:41-51

2. Kellner CH, Husain MM, Knapp RG, McCall WV, Petrides G, Rudorfer MV, Young RC, Sampson S, McClintock SM, Mueller M, Prudic J, Greenberg RM, Weiner RD, Bailine SH, Rosenquist PB, Raza A, Kaliora S, Latoussakis V, Tobias KG, Briggs MC, Liebman LS, Geduldig ET, Teklehaimanot AA, Lisanby SH, Group CPW (2016) Right unilateral ultrabrief pulse ect in geriatric depression: Phase 1 of the pride study. Am $\mathrm{J}$ Psychiatry:appiajp201615081101

3. Haq AU, Sitzmann AF, Goldman ML, Maixner DF, Mickey BJ (2015) Response of depression to electroconvulsive therapy: A meta-analysis of clinical predictors. J Clin Psychiatry 76:1374-1384

4. Nordenskjold A, von Knorring L, Engstrom I (2012) Predictors of the short-term responder rate of electroconvulsive therapy in depressive disorders--a population based study. BMC psychiatry 12:115

5. Krystal AD, Weiner RD (1994) Ect seizure therapeutic adequacy. Convuls Ther 10:153164

6. Sackeim HA, Luber B, Katzman GP, Moeller JR, Prudic J, Devanand DP, Nobler MS (1996) The effects of electroconvulsive therapy on quantitative electroencephalograms. Relationship to clinical outcome. Arch Gen Psychiatry 53:814824

7. Argyelan M, Lencz T, Kaliora S, Sarpal DK, Weissman N, Kingsley PB, Malhotra AK, Petrides G (2016) Subgenual cingulate cortical activity predicts the efficacy of electroconvulsive therapy. TransI Psychiatry 6:e789

8. Redlich R, Opel N, Grotegerd D, Dohm K, Zaremba D, Burger C, Munker S, Muhlmann L, Wahl P, Heindel W, Arolt V, Alferink J, Zwanzger P, Zavorotnyy M, Kugel H, Dannlowski U (2016) Prediction of individual response to electroconvulsive therapy via machine learning on structural magnetic resonance imaging data. JAMA Psychiatry 73:557-564

9. Jiang R, Abbott CC, Jiang T, Du Y, Espinoza R, Narr KL, Wade B, Yu Q, Song M, Lin D, Chen J, Jones T, Argyelan M, Petrides G, Sui J, Calhoun VD (2017) Smri biomarkers predict electroconvulsive treatment outcomes: Accuracy with independent data sets. Neuropsychopharmacology

10. Minelli A, Maffioletti E, Bortolomasi M, Conca A, Zanardini R, Rillosi L, Abate M, Giacopuzzi M, Maina G, Gennarelli M, Bocchio-Chiavetto L (2014) Association between baseline serum vascular endothelial growth factor levels and response to electroconvulsive therapy. Acta Psychiatr Scand 129:461-466

11. Okamoto T, Yoshimura R, Ikenouchi-Sugita A, Hori H, Umene-Nakano W, Inoue $Y$, Ueda N, Nakamura J (2008) Efficacy of electroconvulsive therapy is associated with changing blood levels of homovanillic acid and brain-derived neurotrophic factor (bdnf) in refractory depressed patients: A pilot study. Progress in neuro-psychopharmacology \& biological psychiatry 32:1185-1190

12. Nierenberg AA, DeCecco LM (2001) Definitions of antidepressant treatment response, remission, nonresponse, partial response, and other relevant outcomes: A focus on treatment-resistant depression. J Clin Psychiatry 62 Suppl 16:5-9

13. Hoyer C, Kranaster L, Janke C, Sartorius A (2014) Impact of the anesthetic agents ketamine, etomidate, thiopental, and propofol on seizure parameters and seizure 
quality in electroconvulsive therapy: A retrospective study. Eur Arch Psychiatry Clin Neurosci 264:255-261

14. Kranaster L, Kammerer-Ciernioch J, Hoyer C, Sartorius A (2011) Clinically favourable effects of ketamine as an anaesthetic for electroconvulsive therapy: A retrospective study. Eur Arch Psychiatry Clin Neurosci 261:575-582

15. Kranaster L, Aksay SS, Bumb JM, Janke C, Alonso A, Hoyer C, Zerr I, Schmitz M, Hausner L, Frolich L, Sartorius A (2016) Electroconvulsive therapy selectively enhances amyloid beta 1-42 in the cerebrospinal fluid of patients with major depression: A prospective pilot study. Eur Neuropsychopharmacol 26:1877-1884

16. Kvartsberg $H$, Duits $F H$, Ingelsson $M$, Andreasen N, Ohrfelt A, Andersson K, Brinkmalm G, Lannfelt L, Minthon L, Hansson O, Andreasson U, Teunissen CE, Scheltens P, Van der Flier WM, Zetterberg H, Portelius E, Blennow K (2015) Cerebrospinal fluid levels of the synaptic protein neurogranin correlates with cognitive decline in prodromal alzheimer's disease. Alzheimer's \& dementia : the journal of the Alzheimer's Association 11:1180-1190

17. Bindila L, Lutz B (2016) Extraction and simultaneous quantification of endocannabinoids and endocannabinoid-like lipids in biological tissues. Methods Mol Biol 1412:9-18

18. Kranaster L, Hoyer C, Aksay SS, Bumb JM, Muller N, Zill P, Schwarz MJ, Sartorius A (2017) Antidepressant efficacy of electroconvulsive therapy is associated with a reduction of the innate cellular immune activity in the cerebrospinal fluid in patients with depression. World J Biol Psychiatry:1-11

19. Mattsson N, Insel PS, Palmqvist S, Portelius E, Zetterberg H, Weiner M, Blennow K, Hansson O, Alzheimer's Disease Neuroimaging I (2016) Cerebrospinal fluid tau, neurogranin, and neurofilament light in alzheimer's disease. EMBO Mol Med 8:11841196

20. Serafini G, Adavastro G, Canepa G, Capobianco L, Conigliaro C, Pittaluga F, Murri MB, Valchera A, De Berardis D, Pompili M, Lindqvist D, Brundin L, Amore M (2017) Abnormalities in kynurenine pathway metabolism in treatment-resistant depression and suicidality: A systematic review. CNS \& neurological disorders drug targets 16:440-453

21. Cervenka I, Agudelo LZ, Ruas JL (2017) Kynurenines: Tryptophan's metabolites in exercise, inflammation, and mental health. Science 357

22. Myint AM, Schwarz MJ, Muller N (2012) The role of the kynurenine metabolism in major depression. J Neural Transm (Vienna) 119:245-251

23. Schwieler L, Samuelsson M, Frye MA, Bhat M, Schuppe-Koistinen I, Jungholm O, Johansson AG, Landen M, Sellgren CM, Erhardt S (2016) Electroconvulsive therapy suppresses the neurotoxic branch of the kynurenine pathway in treatment-resistant depressed patients. Journal of neuroinflammation 13:51

24. Guloksuz S, Arts B, Walter S, Drukker M, Rodriguez L, Myint AM, Schwarz MJ, Ponds $R$, van Os J, Kenis G, Rutten BP (2015) The impact of electroconvulsive therapy on the tryptophan-kynurenine metabolic pathway. Brain, behavior, and immunity 48:48-52

25. Muller N (2014) Immunology of major depression. Neuroimmunomodulation 21:123130

26. Kohler CA, Freitas TH, Maes M, de Andrade NQ, Liu CS, Fernandes BS, Stubbs B, Solmi M, Veronese N, Herrmann N, Raison CL, Miller BJ, Lanctot KL, Carvalho AF (2017) Peripheral cytokine and chemokine alterations in depression: A meta-analysis of 82 studies. Acta Psychiatr Scand 135:373-387

27. Lopresti AL, Maker GL, Hood SD, Drummond PD (2014) A review of peripheral biomarkers in major depression: The potential of inflammatory and oxidative stress 
biomarkers. Progress in neuro-psychopharmacology \& biological psychiatry 48:102111

28. Giil LM, Midttun O, Refsum H, Ulvik A, Advani R, Smith AD, Ueland PM (2017) Kynurenine pathway metabolites in alzheimer's disease. Journal of Alzheimer's disease : JAD 60:495-504

29. Fjell AM, Idland AV, Sala-Llonch R, Watne LO, Borza T, Braekhus A, Lona T, Zetterberg H, Blennow K, Wyller TB, Walhovd KB (2017) Neuroinflammation and tau interact with amyloid in predicting sleep problems in aging independently of atrophy. Cereb Cortex:1-11

30. Muller N, Schwarz MJ (2007) The immune-mediated alteration of serotonin and glutamate: Towards an integrated view of depression. Mol Psychiatry 12:988-1000

31. Myint AM, Kim YK (2014) Network beyond ido in psychiatric disorders: Revisiting neurodegeneration hypothesis. Progress in neuro-psychopharmacology \& biological psychiatry 48:304-313

32. Leonard BE, Myint A (2006) Changes in the immune system in depression and dementia: Causal or coincidental effects? Dialogues Clin Neurosci 8:163-174

33. Kranaster L, Hoyer C, Aksay SS, Bumb JM, Leweke FM, Janke C, Thiel M, Lutz B, Bindila L, Sartorius A (2017) Electroconvulsive therapy enhances endocannabinoids in the cerebrospinal fluid of patients with major depression: A preliminary prospective study. Eur Arch Psychiatry Clin Neurosci

34. Smaga I, Bystrowska B, Gawlinski D, Przegalinski E, Filip M (2014) The endocannabinoid/endovanilloid system and depression. Curr Neuropharmacol 12:462474

35. Dinoff A, Herrmann N, Lanctot KL (2017) Ceramides and depression: A systematic review. Journal of affective disorders 213:35-43

36. Jernigan PL, Hoehn RS, Grassme H, Edwards MJ, Muller CP, Kornhuber J, Gulbins E (2015) Sphingolipids in major depression. Neurosignals 23:49-58 


\section{Table}

\begin{tabular}{|l|l|}
\hline Number of included subjects & 12 \\
\hline Age (mean) \pm SD in years (min - max) & $59.0 \pm 21.9(21-83)$ \\
\hline Sex female/male n/n (in \%) & $7 / 5(58.3 / 41.7)$ \\
\hline Type of depression: unipolar/bipolar n/n (in \%) & $9 / 3(75.0 / 25.0)$ \\
\hline Duration of current depressive episode in months, mean \pm SD (min - max) & $18.7 \pm 21.9(1-60)$ \\
\hline Age of onset (mean) \pm SD in years (min - max) & $49.2 \pm 21.8(12-80)$ \\
\hline Duration of illness in years, mean \pm SD (min - max) & $9.9 \pm 9.1(0.5-26)$ \\
\hline Body mass index, mean \pm SD (min - max) & $23.5 \pm 3.5(20-31)$ \\
\hline HDRS, sum score & \\
\hline Baseline HDRS, mean \pm SD (min - max) & $29.9 \pm 6.6(21-41)$ \\
\hline Final HDRS, mean (min - max) & $9.0 \pm 5.2(3-16)$ \\
\hline Mean change \pm SD (min - max) & $20.9(-6-36)(p<0.001)$ \\
\hline Numbers of ECT sessions, mean \pm SD (min - max) & $10.6 \pm 5.0(4-19)$ \\
\hline Days between the two CSF samples, mean \pm SD (min - max) & $35.7 \pm 15.3(17-59)$ \\
\hline Days between last ECT and 2nd sample, mean \pm SD (min - max) & $4.6 \pm 2.4(2-7)$ \\
\hline Switch to bilateral stimulation $n$ (in \%) & $3(25)$ \\
\hline Remitters/Responders/Non-responders ${ }^{*} \mathrm{n} / \mathrm{n} / \mathrm{n}$ (in \%) & $5 / 10 / 2(41.7 / 83.4 / 16.7)$ \\
\hline
\end{tabular}

Table 1: Demographic and clinical features of the patients.

\begin{tabular}{|c|c|c|c|c|}
\hline & $\begin{array}{l}\text { Absolute HDRS } \\
\text { reduction }\end{array}$ & $\begin{array}{l}\text { Relative } \\
\text { HDRS } \\
\text { reduction } \\
\end{array}$ & $\begin{array}{l}\text { Responder } \\
\text { vs. Non- } \\
\text { Responder }\end{array}$ & $\begin{array}{l}\text { No correlation } \\
\text { with outcome }\end{array}$ \\
\hline $\begin{array}{c}\text { Markers of } \\
\text { neurodegeneration }\end{array}$ & $\begin{array}{c}\mathrm{A} \beta 1-40 \\
\text { tau protein } \\
\text { p-tau protein } \\
\mathrm{Ng}\end{array}$ & 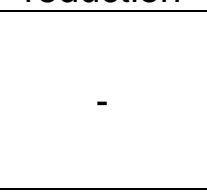 & $\begin{array}{l}\text { tau protein* } \\
\text { p-tau } \\
\text { protein }^{*} \\
\mathrm{Ng}^{*}\end{array}$ & $A \beta 1-42$ \\
\hline Endocannabinoids & AEA & - & - & $\begin{array}{c}2-A G \\
A A\end{array}$ \\
\hline $\begin{array}{l}\text { Elements of the } \\
\text { innate immune } \\
\text { system }\end{array}$ & $\begin{array}{l}\text { SCD14 } \\
\text { IL-6* } \\
\text { Neopterin* } \\
\text { sCD163* }\end{array}$ & sCD14 & sCD163* & $\begin{array}{c}\text { MIF } \\
\text { MCP-1 }\end{array}$ \\
\hline $\begin{array}{l}\text { Metabolies of the } \\
\text { kynurenin pathway }\end{array}$ & $\begin{array}{c}\text { KYN } \\
\text { KYN/TRY } \\
\text { PIC* }^{*} \\
\text { QUIN* }^{*}\end{array}$ & $\begin{array}{c}\mathrm{PIC} \\
\mathrm{KYN} \\
\mathrm{KYNA}^{*} \\
\mathrm{KYN}^{\prime} / \mathrm{TRY}^{*}\end{array}$ & $\begin{array}{c}\mathrm{KYN} \\
\mathrm{KYNA}^{*} \\
\mathrm{PIC}^{*}\end{array}$ & $\begin{array}{l}\text { TRY } \\
3-\mathrm{HK}\end{array}$ \\
\hline Spingholipids & $\begin{array}{c}\text { Total amount of } \\
\text { sphingolipids* } \\
\text { Phosphatidylcholines* }\end{array}$ & - & - & $\begin{array}{c}\text { Ceramide } \\
\text { Sphingomyelin }\end{array}$ \\
\hline sKlotho & - & - & - & sKlotho \\
\hline
\end{tabular}

Table 2: Summary of the results for each category.

${ }^{*}=$ Statistical significance at trend level $(0.1>p>0.05)$ 
Figures

IN PREPARATION 